\title{
Antioxidant modified amphiphilic polymer improves intracellular cryoprotectant delivery and alleviates oxidative stress in HeLa cells
}

\author{
Alexander Chen, Sergio A Mercado and Nigel KH Slater* \\ Department of Chemical Engineering, University of Cambridge, Cambridge, UK
}

\begin{abstract}
The design and synthesis of a dual-function, cell permeating polymer with an antioxidative property is described and its use for the intracellular delivery of the cryoprotectant trehalose into HeLa cells is demonstrated. The polymer, PVitE-25, was created by grafting the water insoluble hydrophobic antioxidant $( \pm)-\alpha$-Tocopherol (vitamin E) onto pendant carboxylate groups of a biocompatible cell permeating polymer, poly (L-lysine iso-phthalamide) (PLP). The modification increased the intracellular delivery efficiency of the polymer and also introduced an antioxidative effect that was able to reduce $85 \%$ of reactive oxygen species (ROS) in HeLa cells incubated with $1 \mathrm{mM}$ hydrogen peroxide $\left(\mathrm{H}_{2} \mathrm{O}_{2}\right)$, as determined by $2^{\prime}, 7^{\prime}$-Dichlorofluorescin diacetate (DCFH-DA) probe. PVitE-25 was also used to load the cropreservative trehalose into HeLa cells prior to freezing such that the level of cell viability measured 48 hours after cell revival was comparable to that observed with a standard $\mathrm{Me}_{2} \mathrm{SO}$-based cryopreservation protocol. This is the first report of a synthetic intracellular delivery system that facilitates the intracellular delivery of the cryoprotectant, trehalose, and mitigates oxidative stress during the freeze thaw cycle of cryopreservation.
\end{abstract}

\section{Introduction}

Cell-based therapy is a rapidly emerging industry and is estimated to be worth around $\$ 5$ billion in the USA alone [1]. Despite wellestablished cryopreservation protocols, generally involving use of $\mathrm{Me}_{2} \mathrm{SO}$, cells remain subject to cryo-damage leading to compromised cell function. Cell damage includes lipid and protein oxidation, which can severely affect cell stability and ability to proliferate [2]. Oxidative stress also induces apoptosis in various cell types, which results in cell loss during cryopreservation [3-6]. Thus, reducing the impact of oxidation during cryopreservation is paramount to enhance cell recovery post freeze/thaw cycles.

To survive long-term storage at low temperatures, cells require treatment with cryoprotective agents, such as dimethyl sulfoxide $\left(\mathrm{Me}_{2} \mathrm{SO}\right)$ or glycerol [7-9], the toxicity of which has been recognized as a critical barrier to further advancement of the field $[7,10]$. Sugars are key non-toxic components in many cryopreservation protocols yet transmembrane transport of sugars is restricted to monosaccharides since disaccharides do not readily diffuse across the plasma membrane [11]. This is a major challenge when high concentrations of disaccharides such as trehalose are needed within cells for viable cryopreservation $[7,12]$. Approaches have been developed to increase the levels of intracellular disaccharides, such as the use of ATP receptor channels or engineered membrane pores [12-14]. Disadvantages of these approaches include the induction of cell death by either necrosis or apoptosis [15].

One effective method to induce disaccharide transport into cells involves the use of amphiphilic polymers that permeabilise cell membranes. For example, polymers have been developed to mimic the membrane permeabilising activity of viral and bacterial peptides [16]. A pH-responsive pseudo-peptide has been previously developed by Eccleston et al. as a delivery system [7]. The polymer, poly (L-lysine iso- phthalamide) or PLP, was able to transport membrane impermeable calcein into the cell cytosol and was well tolerated by mammalian cells in vitro [8]. Various efforts were made to improve the delivery efficiency of the PLP polymer. For example, Chen et al. discovered that by grafting a hydrophobic amino acid as a pendant onto the PLP backbone the intracellular delivery efficiency could be increased $[9,10]$. One such polymer, PP-50, was synthesised by reacting $50 \%$ molar ratio of phenylalanine with free carboxylate of PLP and this polymer has been used to load trehalose into cells to assist cryopreservation $[11,12]$. Notwithstanding, this approach is not able to mitigate the effects of oxidative stress [17-22].

The present study was motivated by the hypothesis that the co-delivery of an anti-oxidant into cells prior to freezing would mitigate oxidative stresses and enable improved cell recovery. $( \pm)$ - $\alpha$ Tocopherol (vitamin E), a natural occurring hydrophobic molecule with antioxidative properties, was chosen as a modifying agent for PLP. Vitamin E is a lipophilic molecule that resides in bio membranes and acts as an extremely effective scavenger for free radicals; reportedly more potent than vitamin $\mathrm{C}$ in preventing lipid peroxidation [2325]. The hypothesis was that vitamin E modified PLP can produce an improved intracellular delivery system for cryopreservation, in part because of the hydrophobicity of vitamin $\mathrm{E}$ and because it is an

Correspondence to: Nigel KH Slater, Department of Chemical Engineering, University of Cambridge, Phillipa Fawcett Drive, West Cambridge Site, Cambridge CB3 0AS, UK, Tel: +44 (0) 1223 762953; Fax: +44 (0) 1223 334796; E-mail:nkhs2@cam.ac.uk

Key words: intracellular delivery, antioxidative polymer, functional biopolymer, biocompatible polymer

Received: September 20, 2017; Accepted: October 23, 2017; Published: October 26,2017 
effective antioxidant. Furthermore, vitamin E could not be delivered independently into cells since it is only sparingly soluble in aqueous environments.

The resulting aqueous soluble polymer, PVitE-25, was tested for its effectiveness by observation of its intracellular delivery efficiency for membrane impermeable calcein and also by measurements of intracellular free-radical responsive fluorescence of 2',7'-Dichlorofluorescin diacetate (DCFH-DA) assay compared to unmodified PLP polymer [16-18]. Additionally, as a proof of concept, cryopreservation was chosen as an application to test the effectiveness of the PVitE-25 polymer for the following two reasons. Firstly, an intracellular delivery system is required for trehalose, a naturally occurring membrane impermeable disaccharide with cryoprotective properties, as it has to be present on both sides of the cell membrane in order to protect cells through vitrification during the freeze thaw cycle [3,19-21]. Secondly, oxidative stress was observed in the freezing of various cell types and led to different levels of apoptotic cell death [4-6,22].

\section{Materials and methods}

\section{Materials}

L-lysine methyl ester dihydrochloride was purchased from Tokyo Chemical Industry UKLtd (Oxford, UK). Acetone, potassium carbonate, $( \pm)$ - $\alpha$-Tocopherol synthetic (Vitamin-E), dimethylformamide (DMF), 4-dimethylaminopyridine (DMAP), N,N'-dicyclohexylcarbodiimide (DCC), isophthaloyl chloride, saponin, ethanol, D-(+)-trehalose dehydrate, calcein, hydrogen peroxide $\left(\mathrm{H}_{2} \mathrm{O}_{2}\right), 2^{\prime}, 7^{\prime}$ - dichlorofluorescin diacetate (DCFH-DA), disodium hydrogen phosphate, and sodium phosphate were purchased from Sigma Aldrich (Dorset, UK). Dimethyl sulfoxide $\left(\mathrm{Me}_{2} \mathrm{SO}\right)$, sodium hydroxide, and $\mathrm{Gibco}^{\mathrm{TM}}$ trypsinEDTA $(0.05 \%)$ were purchased from Fisher Scientific (Loughborough, UK). Visking dialysis tubing (12-14 kDa cut off) was purchased from Medicell Membranes Ltd (London, UK). Dulbecco's Modified Eagle Medium (DMEM), penicillin streptomycin (Pen/Strep 10,000 U/mL), Hoechst 33342, and propidium iodide (PI) were purchased from Life Technologies (Paisley, UK). Fetal bovine serum (FBS) and phosphate buffer saline (PBS) were purchased from Sigma Aldrich (Dorset, UK). CellTiter $96^{\circ}$ AQueous One Solution Cell Proliferation Assay (MTS) was purchased from Promega (Southampton, UK). FITC AnnexinV Apoptosis Detection Kit was purchased from BD, UK (Oxford, UK). All chemicals were purchased from Sigma Aldrich (Dorset, UK) and biochemical reagents from Life Technologies (Paisley, UK) if not specified otherwise.

\section{Polymer synthesis and characterisation}

Poly (L-lysine iso-phthalamide) (PLP) was synthesised as previously reported by Eccleston et al. $[7,8]$. Vitamin-E was grafted onto PLP backbone at $25 \%$ molar ratio of available carboxylate group

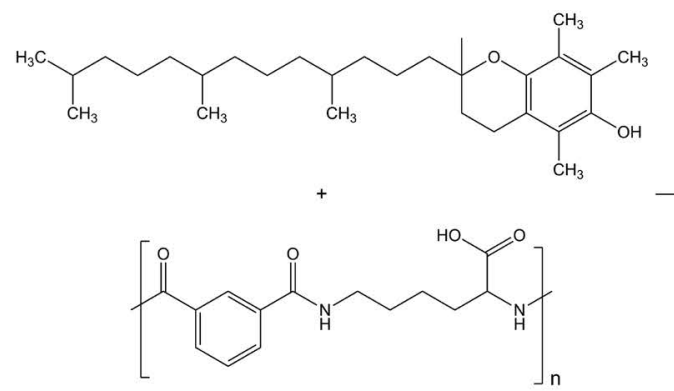

through esterification with standard DCC coupling reaction [23]. The reaction scheme of PVitE-25 synthesis is depicted in Figure 1.

\section{H $^{1}$ NMR}

Polymers were dissolved in $\mathrm{Me}_{2} \mathrm{SO}-\mathrm{d} 6$ and characterised by Bucker Advance $500 \mathrm{MHz}$ NMR spectroscopy (Bruker Biospin GmbH, Germany).

\section{Cell culture}

HeLa adherent epithelial cells (human cervical cancer cells) were grown in DMEM media supplemented with $10 \%$ (v/v) FBS, $2 \mathrm{mM}$ L-glutamine, $100 \mathrm{U} / \mathrm{mL}$ pen/strep unless specified otherwise. HeLa cells were trypsinised using trypsin-EDTA and maintained in a humidified incubator with $5 \% \mathrm{CO}_{2}$ at $37^{\circ} \mathrm{C}$.

\section{Confocal microscopy}

Intracellular calcein distribution: HeLa cells were incubated in supplemented DMEM for 48 hours before the experiment in a 4 -well $1.8 \mathrm{~cm}^{2}$ chambered cover glass system (Nunc, UK) at $5 \times 10^{4}$ cells/well and $500 \mu \mathrm{L} /$ well. Cells were first washed twice with PBS and incubated with phenol red- and FBS-free DMEM in the presence or absence of 2 $\mathrm{mM}$ calcein for 24 hours with or without PVitE-25 polymer. Cells were then washed twice with PBS and incubated with $5 \mu \mathrm{g} / \mathrm{mL}$ Hoecht 33342 and $5 \mu \mathrm{g} / \mathrm{mL}$ PI in phenol red- and FBS- free DMEM for 20 minutes. Cells were washed again twice with PBS before phenol red-free DMEM was added to each well and imaged under confocal microscopy.

Cellular ROS measurement using DCFH-DA: HeLa cells were incubated in supplemented DMEM for 48 hours before the experiment in 4 -well $1.8 \mathrm{~cm}^{2}$ chambered cover glass system (Nunc, UK) at $5 \times$ $10^{4}$ cells/well and $500 \mu \mathrm{L} /$ well. Cells were washed twice with PBS and incubated with phenol red- and FBS-free DMEM in the presence or absence of $1 \mathrm{mM} \mathrm{H} \mathrm{H}_{2}$ for 2 hours with or without polymer. Cells were then washed twice with PBS and incubated with $10 \mu \mathrm{M} \mathrm{DCFH}-$ $\mathrm{DA}, 5 \mu \mathrm{g} / \mathrm{mL}$ Hoechst 33342, $5 \mu \mathrm{g} / \mathrm{mL}$ PI in phenol red- and FBS- free DMEM for 20 minutes. Cells were washed again twice with PBS and DMEM was added to each well and imaged by confocal microscopy.

Image were processed using Image J. Fluorescent intensity per cell was calculated by the dividing total image fluorescent intensity by the total cell number. Cell numbers were determined by counting cells that had Hoechst 33342 stained nuclei.

\section{Intracellular delivery efficiency of polymers}

Calcein (a membrane impermeable fluorophore) was used as a tracer molecule to monitor the effect of polymer intracellular delivery efficiency. HeLa cells were seeded in 24 -well plates at $5 \times 10^{4}$ cells $/ \mathrm{mL}$ in $0.5 \mathrm{~mL}$ supplemented DMEM to full confluence in 24 hours followed by incubation with $0.22 \mu \mathrm{m}$ filter- sterilised supplemented media and 2 $\mathrm{mM}$ calcein in the presence or absence of $1 \mathrm{mg} / \mathrm{mL}$ PVitE-25 polymer.

Figure 1. Reaction scheme of PVitE-25 synthesis. Standard DCC esterification using DMAP as catalyst to graft ( \pm )- $\alpha$-Tocopherol onto the PLP polymer backbone. 


\section{Cytotoxicity assays}

MTS assay: HeLa cells were seeded in 96-well plates at $5 \times 10^{3}$ cells/well in $100 \mu \mathrm{L}$ of supplemented media for 48 hours. Cells were then incubated with phenol red free DMEM supplemented FBS with or without PVitE-25 for 24 or 48 hours at different concentrations. The MTS assay was performed according to the manufacturer's recommendation. Data was collected at $480 \mathrm{~nm}$ with a SPECTROstar Nano plate reader (BMG Labtech Ltd., UK).

AnnexinV/PI apoptosis assay: HeLa cells were seeded in 24well plates at $5 \times 10^{4}$ cells/well in $500 \mu \mathrm{L}$ supplemented media for 48 hours. Cells were then incubated with supplemented media with or without PVitE-25 for 24 and 48 hours. Cells were then washed with PBS twice, trypsinised, and then re-suspended in phenol red-free DMEM containing FITC-AnnxinV and PI. Untreated cells were used as a negative control. HeLa cells incubated with $1 \%$ saponin in DMEM for 10 minutes were used as a positive control for apoptosis. The apoptosis assay was then performed with flow cytometry to determine the cytotoxicity of the polymers.

\section{Cryopreservation}

HeLa cells were seeded in 6 -well plates at $5 \times 10^{5}$ cells $/ \mathrm{mL}$ in 1 $\mathrm{mL}$ of supplemented media for 24 hours. Cells were then incubated with phenol red free DMEM media supplemented with 10\% FBS with or without $200 \mathrm{mM}$ of trehalose in the presence or absence of $1 \mathrm{mg} /$ $\mathrm{mL}$ PVitE-25 for 4 hours. After the incubation, cells were washed three times with PBS, trypsinised and spun down. Cells were then resuspended in $10 \%(\mathrm{v} / \mathrm{v}) \mathrm{Me}_{2} \mathrm{SO}$ in $\mathrm{FBS}$ or $100 \mathrm{mM}$ trehalose in $10 \%(\mathrm{v} / \mathrm{v})$ phenol red free DMEM media in FBS at $10^{6}$ cells/well. The solution containing cells was transferred into cryovials and cooled at $1{ }^{\circ} \mathrm{C} / \mathrm{min}$ in $\mathrm{Mr}$. Frosty container in a $-80^{\circ} \mathrm{C}$ freezer overnight. The vials were then transferred into a liquid nitrogen cell bank and stored for at least 3 days before thawing. Experiments were performed in triplicates.

During the thawing process, each vial of $1 \mathrm{~mL}$ HeLa cells were diluted with $9 \mathrm{~mL}$ of supplemented DMEM and seeded in 96-well plates. Metabolic activities were assessed by MTS assay 24 and 48 hours after thawing. For the 48-hour timing, media was changed with supplemented DMEM 24 hours after thawing. MTS assay was performed as manufacturer's recommendation. Experiments were performed in six repeats.

\section{Statistical analysis}

All data are reported as the mean \pm standard deviation from at least three independent measurements. The statistical significance in mean values between two groups was analysed using a one-way ANOVA followed by a Tukey's test for multiple comparisons. The tests were analysed using GraphPad Prism (GraphPad Software, US). A p-value of $<0.05$ was taken as statistically significant.

\section{Results}

\section{Polymer synthesis and characterisation}

The structure of PVitE-25 was confirmed by $\mathrm{H}^{1} \mathrm{NMR}$ to be different from the PLP backbone (Figure S1). Triplet peaks at $\delta=1.97$, $2.01,2.03$ indicated the nine protons on the three methyl groups on the benzene ring of $( \pm$ )- $\alpha$-Tocopherol (Vitamin E) and peaks at $\delta=4.33$ represented the protons on the beta carbon of the L-lysine on the PLP backbone. The grafting percentage of PVitE- 25 was calculated to be $18.93 \%$ by quantifying integrated area of the $\mathrm{H}^{1} \mathrm{NMR}$ peaks mentioned before (Figure S1).

\section{Cytotoxicity of PVitE-25}

The metabolic activity of HeLa cells with 24 and 48 hours of polymer incubation at different concentrations was maintained at a similar level relative to the untreated control (Figure 2A). Induced apoptosis and membrane integrity were also tested by commercially available Annexin V apoptosis kit with PI staining. As shown in Figure $2 \mathrm{~B}$, the Annexin $\mathrm{V}$ fluorescent intensities of cells incubated with polymer for 24 hours were similar to the negative control, proving that polymer induced minimal apoptotic activity in HeLa cells postpolymer incubation at the tested concentration. PI staining showed that the membrane integrity of cells incubated with polymer for 24 hours were relatively intact compared to the negative control (Figure 2C). Incubation for 48 hours with the polymer produced a slightly higher Annexin V and PI signal compared to the negative control. These three experiments indicated that PVitE-25 is well tolerated by HeLa cells up to $2 \mathrm{mg} / \mathrm{mL}$ with a 24 -hour incubation periods.

\section{Intracellular delivery efficiency of PVitE-25}

PVitE-25 increased intracellular calcein by $50.5 \pm 11.3 \%, 89.3 \pm$ $0.9 \%$, and $108.1 \pm 1.6 \%$ respectively at $0.5,1$, and $2 \mathrm{mg} / \mathrm{mL}$ compared to calcein only (Figure $3 \mathrm{~A}, \mathrm{p}<0.05$ ). The intracellular calcein concentration also increased in a PVitE-25 concentration-dependent manner. The kinetics of calcein intake were also characterised by flow cytometry and increased in a time-dependent manner (Figure 3B). The endosomal escape characteristic of PVitE-25 was assessed by confocal microscopy to determine calcein distribution in the cell cytosolic space. As shown in Figure 4, calcein was evenly distributed in the cell cytosol after 6-hr incubation with PVitE-25 compared to calcein alone.

\section{Anti-oxidative characteristic of PVitE25}

To determine whether the polymer shows anti-oxidative property, a free radical reactive dye, DCFH- DA, was used to probe for ROS level. Incubation with $\mathrm{H}_{2} \mathrm{O}_{2}(1 \mathrm{mM})$ was used to induce ROS production in HeLa cells in this study. As shown in Figure 5A, hydrogen peroxide (1 $\mathrm{mM}$ ) was able to induce a significant fluorescent signal in HeLa cells and could be observed under confocal microscopy. When the cells were pre-incubated with PLP before the ROS induction, the DCFH-DA fluorescence was decreased by $14.0 \pm 9.2 \%(\mathrm{p}>0.05$, ns). However, when HeLa cells were incubated with hydrogen peroxide in the presence of PVitE-25 $(1 \mathrm{mg} / \mathrm{mL})$ for 2 hours, the amount DCFH-DA fluorescent signal was significantly reduced by $85.4 \pm 7.3 \%$ (Figure $5 B, p<0.05$ ).

\section{Cell viability post-cryopreservation}

The viability of HeLa cells after different cryopreservation protocols was determined by MTS assay 24 and 48 hours after reconstitution with supplemented DMEM. As shown in Figure 6, cells preserved using PVitE-25 combined with the trehalose treatment protocol reached $82.7 \pm 5.5 \%$ viability 24 hours after thawing compared to the standard $\mathrm{Me}_{2} \mathrm{SO}$ preservation method. Additionally, cells preserved with the PVitE-25-based protocol were able to reach comparable metabolic activity 48 hours after thawing as of the standard $\mathrm{Me}_{2} \mathrm{SO}$ protocol while polymer alone $(10.9 \pm 2.0 \%)$ and unmodified polymer backbone $(2.5 \pm$ $1.5 \%)$ failed to protect cells post-thawing.

\section{Discussion}

Poly (L-lysine iso-phthalamide) was developed by Eccleston et al. as a $\mathrm{pH}$ responsive, biocompatible pseudo-peptide intracellular delivery system $[7,8]$. The polymer increases the intracellular delivery by interacting with the cell membrane and facilitating endosomal escape $[9,12]$. The intracellular delivery efficiency of the PLP polymer was shown 


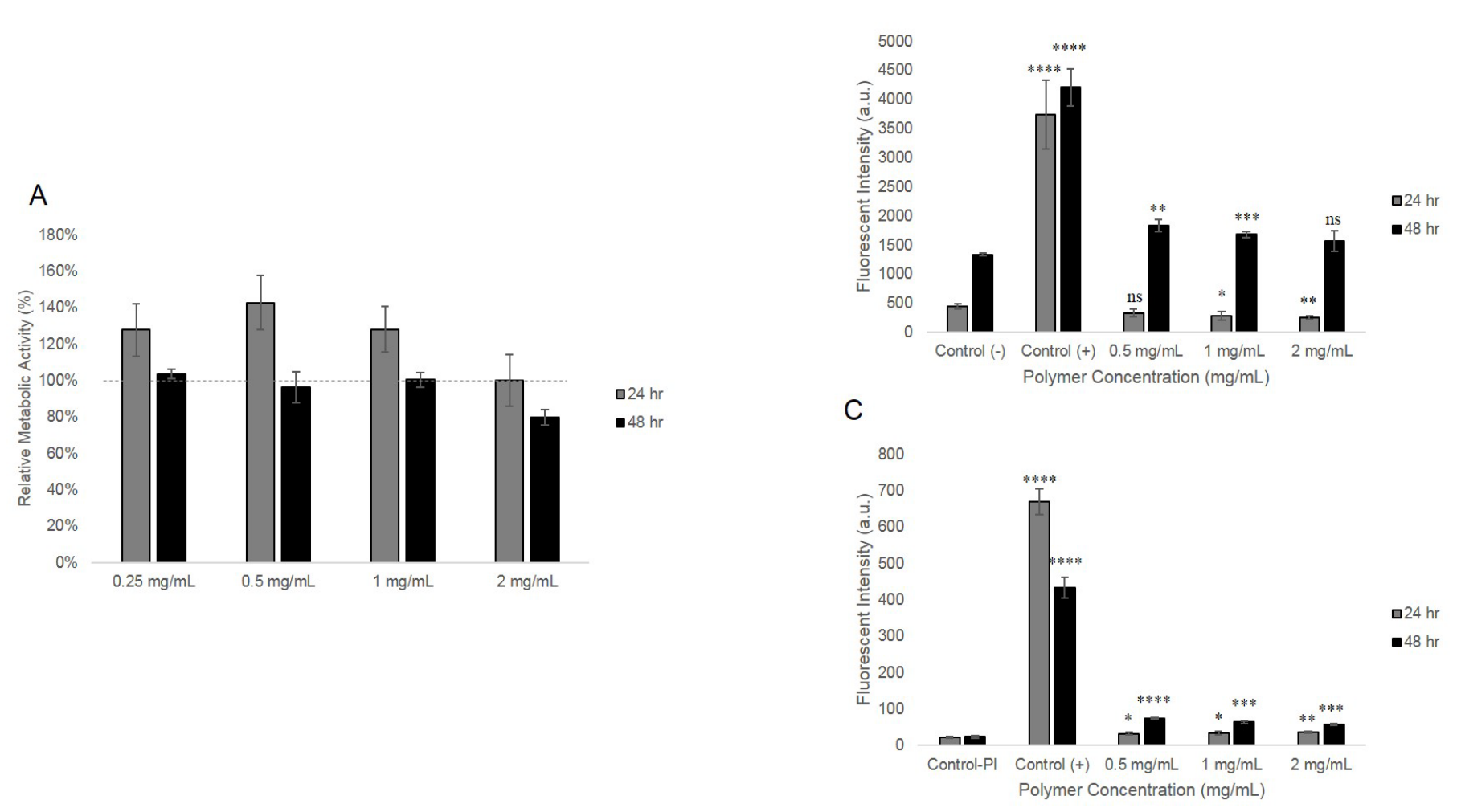

Figure 2. The cytotoxicity of PVitE-25 at different incubation period and concentration. A) Metabolic activity of HeLa cells after 24- and 48-hour incubation with PVitE-25 determined by MTS assay. B) Annexin V staining of HeLa cells after 24- and 48- hour incubation with PVitE-25 polymer at different concentrations. Control (-) and control (+) were untreated cells and cells incubated with $1 \%$ saponin for 10 minutes. C) PI staining of HeLa cells after 24- and 48- hour incubation with PVitE-25 polymer at different concentrations. Control (-) and control (+) were untreated cells and cells incubated with $1 \%$ saponin for 10 minutes.
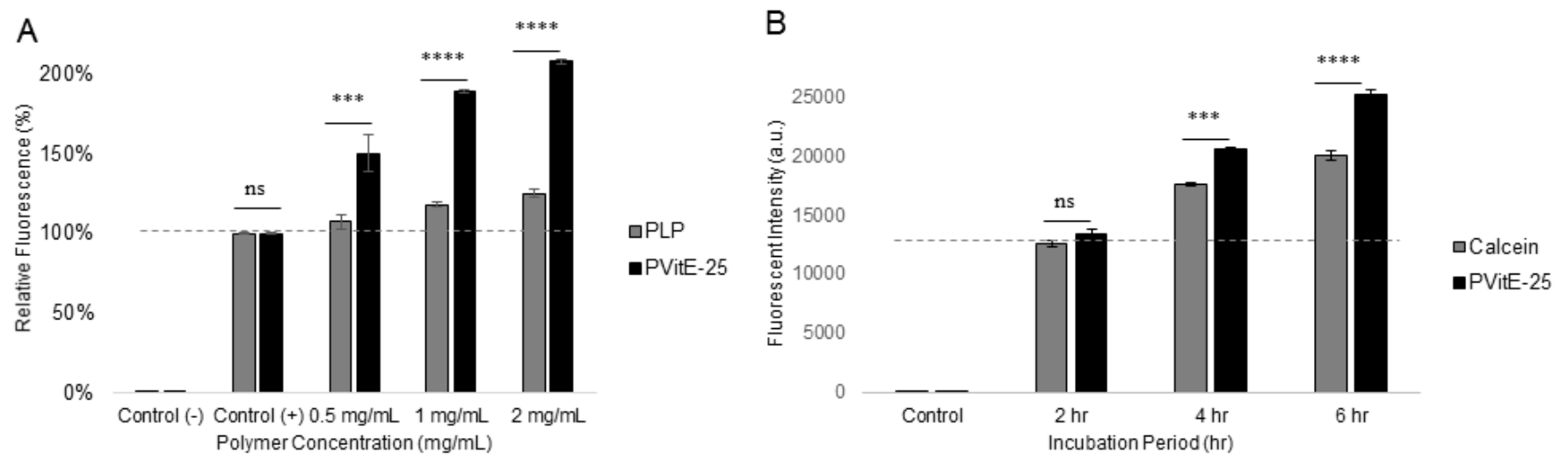

Figure 3. A) Intracellular delivery of calcein by co-incubation with PVitE-25 or PLP for 24 hours at different polymer concentration. B) Intracellular delivery of calcein at different incubation length with $1 \mathrm{mg} / \mathrm{mL}$ of PVitE-25.

to be significantly increased by modification with hydrophobic amino acid $[9,10]$. In this study, cryopreservation was used as a model application and the cell permeating polymer has specifically designed to mitigate oxidative stress. The polymer delivery efficiency was increased and the anti-oxidative function was effective. Along with trehalose the PVitE-25 polymer provided an effective cryopreservation protocol for HeLa cells.

PVitE-25 polymer was synthesised with a standard DCC esterification reaction (Figure 1). From the NMR spectrum PVitE-25 had additional peaks at chemical shifts before $4 \mathrm{ppm}$, which corresponds to three $-\mathrm{CH}_{3}$ groups on the benzene ring and the long hydrocarbon chain of vitamin E molecules (Supplementary Figure S1). Since vitamin
E, like PLP, contains a ring structure, the high chemical shift regions that are usually used for determining the number of monomers would no longer be an accurate measure. Therefore, the single hydrogen on beta carbon of L-lysine in PLP $(\delta=4.33)$ was used instead. The grafting percentage was calculated by comparing the integrated area of the two peaks at $\delta=2.01$ and $\delta=4.33$ respectively with a 9:1 ratio. The grafting percentage was calculated to be $18.93 \%$ which translates to a $75.72 \%$ reaction efficiency. There were no observed stability issues with the polymer after dialysis and lyophilisation [23].

As shown in Figure 2A, PVitE-25 was well tolerated by HeLa cells up to the maximum solubility of $2 \mathrm{mg} / \mathrm{mL}$ according to the MTS 


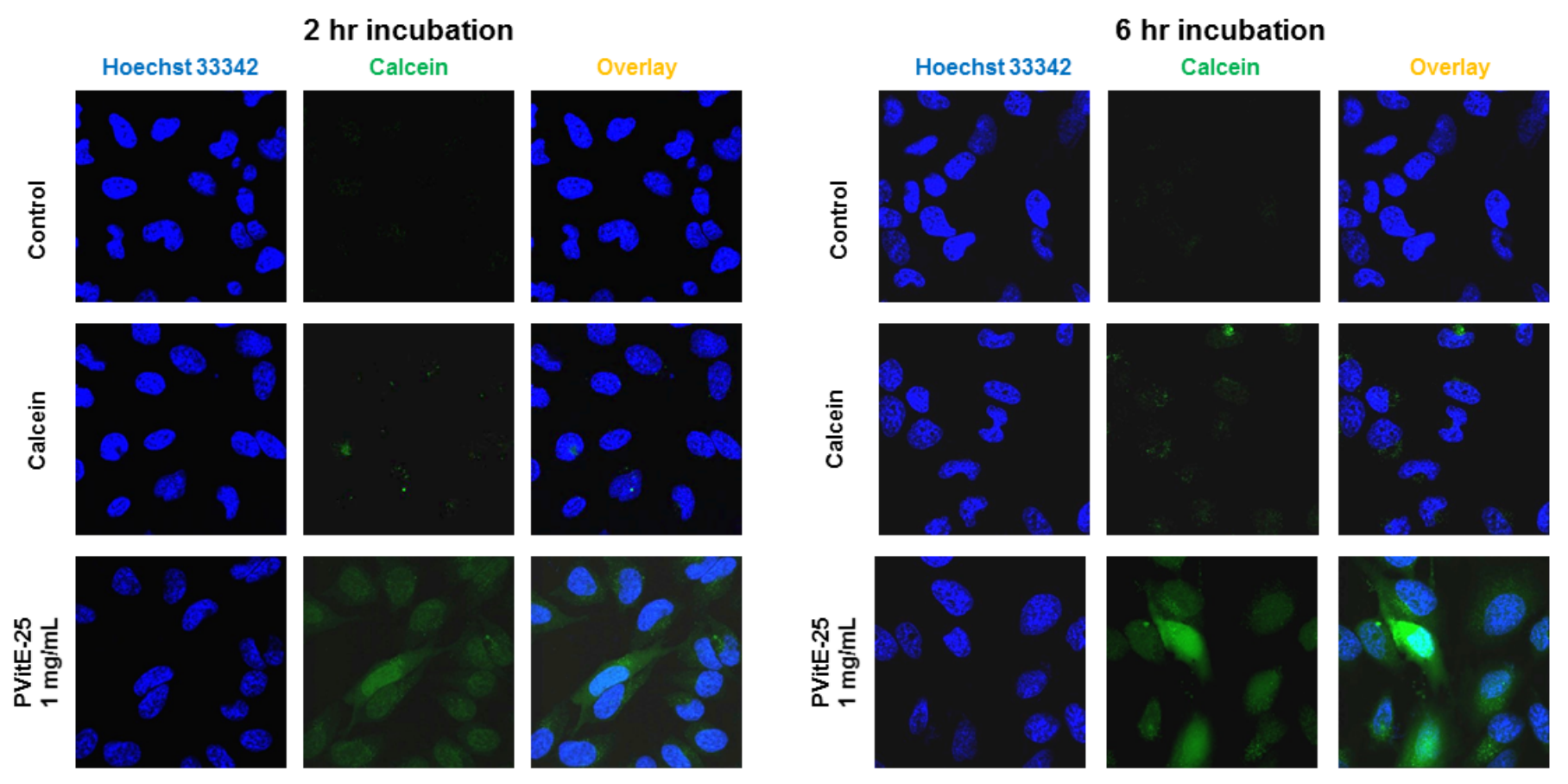

Figure 4. Confocal image of HeLa cells after co-incubation with calcein and PVitE-25. Calcein distribution was used to assess endosomal escape efficiency of PVitE-25. Cell nuclei were stained with Hoechst 33342 (blue). A) 2-hour incubation. B) 4-hour incubation.
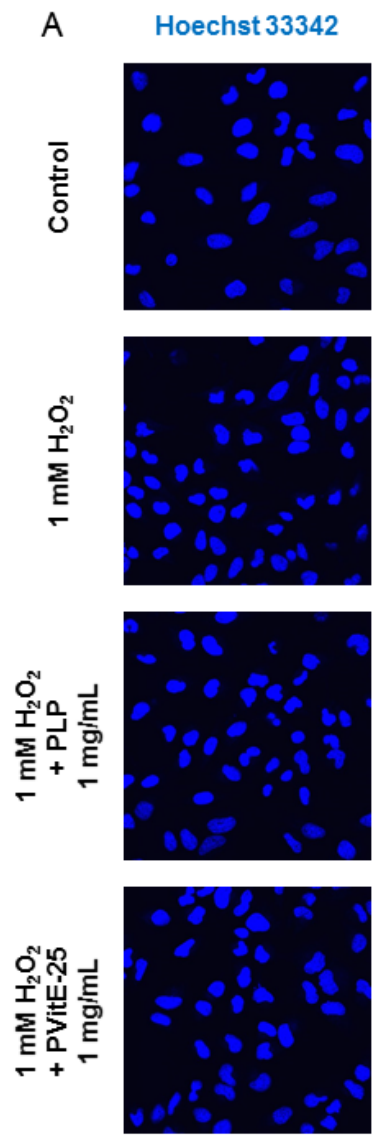

DCF
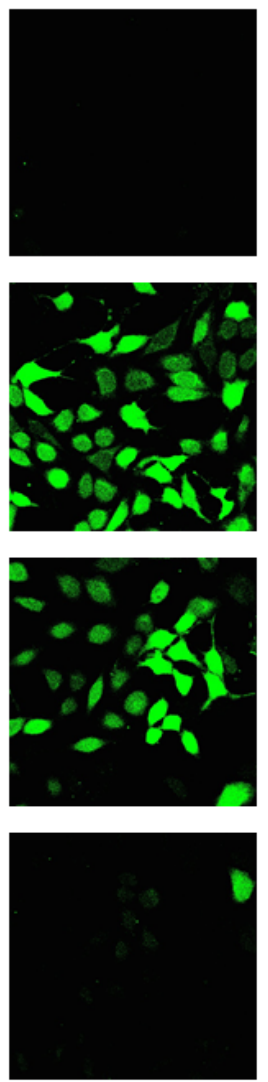

Overlay
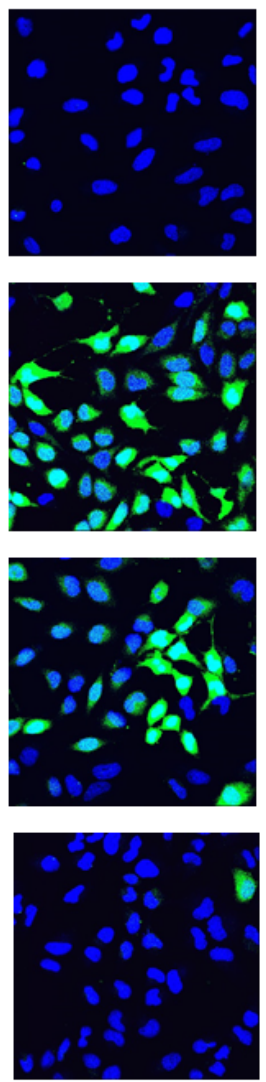

B

60000

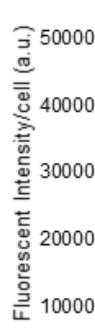

Contro

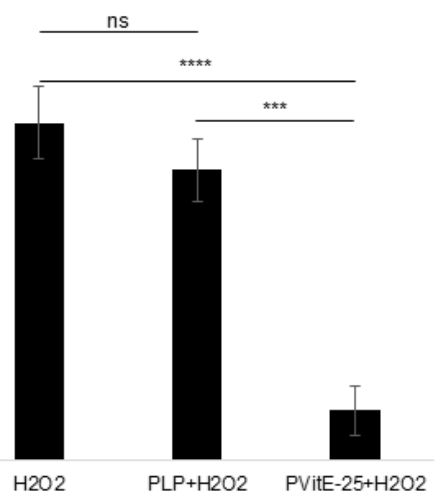

Figure 5. A) Confocal images of HeLa cells incubated with ROS-reactive dye DCFH-DA to assess the level of ROS. The green fluorescent represents DCF which was oxidised from DCFH. Higher fluorescent level corresponds to higher ROS levels. ROS production was induced by incubation with $1 \mathrm{mM} \mathrm{H} 2 \mathrm{O} 2$ for 2 hours in the presence of PVitE-25 or PLP. B) Quantification of cellular ROS level using DCF fluorescent intensity by image processing with Image $\mathrm{J}(\mathrm{n}=3)$. 


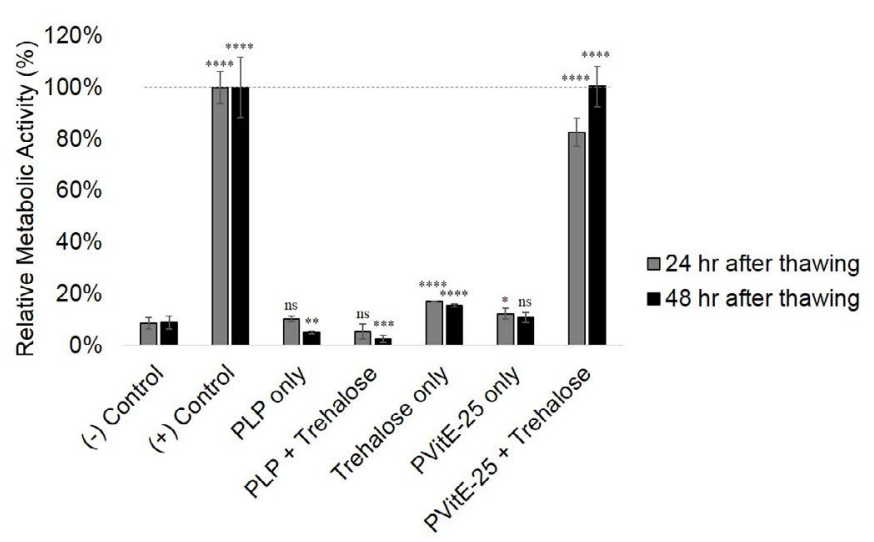

Figure 6. Metabolic activity of HeLa cells 24 and 48 hours after reconstitution from cryostorage determined by MTS assay. HeLa cells were treated with different cryopreservation protocols. Untreated HeLa cells were used as a reference while positive control was Me2SO -based protocol and negative control was frozen cells without cryoprotectant. Cryopreservation was done in triplicates and MTS was done in six repeats.

result. For polymer concentrations below $1 \mathrm{mg} / \mathrm{mL}$, HeLa cells retained higher or similar levels of metabolic activity compared to untreated cells. It was only with $2 \mathrm{mg} / \mathrm{mL}$ and 48 -hour incubation that the HeLa cell metabolic activity fell to $78.94 \% \pm 4.33 \%$ of that of untreated cells. The membrane integrity determined by PI staining of HeLa cells incubated with PVitE- 25 at $2 \mathrm{mg} / \mathrm{mL}$ for 24 hours was also relatively intact compared to saponin, a strong cell permeabilising agent, treated cells (Figure 2C) [24]. The PI fluorescence of HeLa cells after 48-hour incubation with PVitE-25 was, however, increased by on average 1.5fold across different concentrations of PVitE-25 compared to 17-fold of the positive control with saponin. Additionally, the level of induced apoptosis was increased in HeLa cells after 48-hour incubation period with PVitE-25 compared to untreated cells (Figure 2B). The increased signal was on average $27 \%$ across different concentrations of PVitE-25 compared to the negative control of untreated cells, whereas the positive control with saponin increased by 15 - folds. These results suggest that PVitE-25 was well tolerated by HeLa cells with a 24 -hour incubation period at $2 \mathrm{mg} / \mathrm{mL}$. The results from for PVitE- 25 with 48 hour incubation period should not prevent the polymer from being used in the biological system as our cryopreservation protocol used 4 -hour period with polymer concentration of $1 \mathrm{mg} / \mathrm{mL}$.

Vitamin E was used as a hydrophobic modifier to increase PLP delivery efficiency. Vitamin $\mathrm{E}$ is water insoluble and it is more hydrophobic than phenylalanine, therefore the selected grafting percentage for PVitE- 25 was $25 \%$ instead of the $50 \%$ molar ratio reported previously for amino acid modification [16]. As shown in Figure 3A, PVitE-25 was significantly more efficient in the intracellular delivery of calcein with 24 -hour incubation. It was able to achieve a two-fold increase in intracellular calcein at $2 \mathrm{mg} / \mathrm{mL}$ after 24 hours compared to the same concentration of PLP $(\mathrm{p}<0.05)$. The reason for the increase in intracellular delivery could be the increased polymer-membrane interaction cause by the hydrophobic vitamin E modification, like hydrophobic amino acids, which increases the membrane permeability to small molecules and enhanced endosomal escape $[9,10]$.

For delivery systems, like PLP, that utilise the endosomal pathway, endosomal escape is a key characteristic the system should possess to avoid molecules becoming entrapped in the endosome and degraded. Chen et al. proved that PLP and its derivatives were able to facilitate endosomal escape of calcein in HeLa cells and distribute the dye evenly in the cell cytosol [9]. Furthermore, Mercado et al. showed that the phenylalanine modified PLP, PP-50, utilises the endosomal pathway as an intracellular delivery route [12]. Therefore, the PLP-derived PVitE-25 would have to ensure the escape of the cargo before its degradation. As shown in Figure 4B, PVitE-25 was able to facilitate the escape of calcein to the cytosol in HeLa cells, as indicated by the green fluorescence evenly distributed within the cells. These two assessments strongly suggested that PVitE- 25 could be used as a more effective intracellular delivery system over PLP.

Multifunctionality is not a new idea in drug delivery and various designs have utilised this idea to enhance the efficiency of drug delivery systems such as incorporating site specific delivery with antibody or magnetism with metal ions [25]. In this study, the first intracellular delivery system with an effective antioxidative characteristic is reported. The design was deliberately made to alleviate the adverse oxidative stress that occurs with different environmental- or drug-related stimuli, such as occur during cryopreservation. As shown in Figure 5, PLP polymer provided minimal $(14.0 \pm 9.2 \%$, $\mathrm{p}>0.05)$ protection against $\mathrm{H}_{2} \mathrm{O}_{2}$ induced $\mathrm{ROS}$ in HeLa cells. Incubation with PVitE-25, on the other hand, provided significant protection $(85.4 \pm 7.3 \%$ reduction, $\mathrm{p}<0.05$ ) against $\mathrm{H}_{2} \mathrm{O}_{2}$ induced ROS in HeLa cells compared to the positive control. The result suggested that the vitamin $\mathrm{E}$ moiety retains its antioxidative activity when incorporated into PVitE-25 and is able to mitigate oxidative stress within the cell cytoplasm.

The effectiveness of PVitE-25 was further tested in the cryopreservation of HeLa cells. In this study, PVitE-25 was used in conjunction with trehalose as a cryostorage protocol to compare with the standard $\mathrm{Me}_{2} \mathrm{SO}$ protocol. As shown in Figure 6, 24 hours after reconstitution, the relative metabolic activity of HeLa cells using the PVitE-25/trehalose protocol has $82.7 \pm 5.5 \%$ viability compared to untreated and unfrozen HeLa cells. Furthermore, relative metabolic activity of cells 48 hours after reconstitution using the PVitE-25/ trehalose protocol $(100.3 \pm 7.9 \%)$ is comparable to both the $\mathrm{Me}_{2} \mathrm{SO}$ protocol and untreated and unfrozen HeLa cells. It is believed that the successful cryostorage was achieved for two reasons: Firstly, PVitE-25 delivered the membrane impermeable trehalose intracellularly and enabled trehalose to be an effective cryoprotectant [20,21]. Secondly, the antioxidative property of PVitE-25 acted to alleviate the adverse effect ROS caused to cells during the freeze thaw cycle and improve the survival of cells undergoing cryopreservation [6,22]. The cryopreservation result highlighted the successful development of the first synthetic cell permeating polymer system with an antioxidative property and the potential for it be an effective system for other biomedical applications.

\section{Conclusion}

This study introduced a novel design and development of a biocompatible cell permeating polymer with an antioxidative property. The modification of PLP using vitamin E provided a functionally active aqueous soluble form of the antioxidant that retained the ability to enhance the transport of hydrophilic molecules such as trehalose into cells while retaining biocompatibility and the ability to remove $85 \%$ of reactive oxygen species (ROS) in HeLa cells incubated with $1 \mathrm{mM}$ hydrogen peroxide. The modification introduced an effective antioxidative activity that was able to protect HeLa cells from $1 \mathrm{mM}$ $\mathrm{H}_{2} \mathrm{O}_{2}$ challenge. The resulting PVitE-25 polymer was further assessed for the cryopreservation of HeLa cells. When the polymer was used in conjunction with trehalose as a protocol to cryopreserve HeLa cells, it was as effective as the $\mathrm{Me}_{2} \mathrm{SO}$ standard protocol judging by the metabolic activity of the reconstituted cells. This result highlighted the 
effectiveness of PVitE-25 as a dual functional delivery system and its viability in biomedical applications.

\section{Acknowledgement}

This work was supported by Marie Curie Actions under EU FP7 Initial Training Network SNAL608184. The author with to thank Dr. Meng Lu at the Department of Chemical Engineering and Biotechnology, Cambridge University for valuable discussion and kindly provided the DCFH-DA.

\section{References}

1. Ram-Liebig G, Bednarz J, Stuerzebecher B, Fahlenkamp D, Barbagli G, et al. (2015) Regulatory challenges for autologous tissue engineered products on their way from bench to bedside in Europe. Adv Drug Deliv Rev 82-83: 181-191. [Crossref]

2. Best BP1 (2015) Cryoprotectant Toxicity: Facts, Issues, and Questions. Rejuvenation Res 18: 422-436. [Crossref]

3. Wolkers WF, Walker NJ, Tablin F, Crowe JH (2001) Human Platelets Loaded with Trehalose Survive Freeze-Drying. Cryobiology 42: 79-87. [Crossref]

4. Zhang D, Ren L, Chen GQ, Zhang J, Reed BM, et al. (2015) ROS-induced oxidative stress and apoptosis-like event directly affect the cell viability of cryopreserved embryogenic callus in Agapanthus praecox. Plant Cell Rep 34: 1499-1513. [Crossref]

5. Bilodeau JF, Blanchette S, Gagnon C, Sirard MA (2001) Thiols prevent H2O2mediated loss of sperm motility in cryopreserved bull semen. Theriogenology 56: 275 286. [Crossref]

6. Zhang JM, Wang HC, Wang HX, Ruan LH, Zhang YM, Li JT, et al. (2013) Oxidative stress and activities of caspase- $8,-9$, and -3 are involved in cryopreservation-induced apoptosis in granulosa cells. Eur J Obstet Gynecol Reprod Biol 166: 52-55.

7. Eccleston ME, Kuiper M, Gilchrist FM, Slater NK (2000) pH-responsive pseudopeptides for cell membrane disruption. J Control Release 69: 297-307. [Crossref]

8. Eccleston ME, Williams SL, Yue Z, Chen R, Lee CK, Anikina E, et al. (2005) Design and In-vitro Testing of Effective Poly(1-Lysine Iso-Phthalamide) Based Drug Targeting Systems for Solid Tumours. Food Bioprod Process 83: 141-146.

9. Chen R, Khormaee S, Eccleston ME, Slater NK (2009) The role of hydrophobic amino acid grafts in the enhancement of membrane-disruptive activity of $\mathrm{pH}$-responsive pseudo-peptides. Biomaterials 30: 1954-1961. [Crossref]

10. Khormaee S, Chen R, Park JK, Slater NK (2010) The Influence of Aromatic SideChains on the Aqueous Properties of pH-Sensitive Poly(L-lysine iso-phthalamide) Derivatives. J Biomater Sci Polym Ed 21: 1573-1588. [Crossref]
11. Lynch AL, Chen R, Slater NK (2011) PH-responsive polymers for trehalose loading and desiccation protection of human red blood cells. Biomaterials 32: 4443-4449. [Crossref]

12. Mercado SA, Orellana-Tavra C, Chen A, Slater NK (2016) The intracellular fate of an amphipathic $\mathrm{pH}$-responsive polymer: Key characteristics towards drug delivery. Mater Sci Eng C Mater Biol Appl 69: 1051-1057. [Crossref]

13. Tappel AL (1962) Vitamin E as the Biological Lipid Antioxidant. Vitam Horm 20 493-510

14. Burton GW, Traber MG (1990) Vitamin E: antioxidant activity, biokinetics, and bioavailability. Annu Rev Nutr 10: 357-382. [crossref]

15. Packer JE, Slater TF, Willson RL (1979) Direct observation of a free radical interaction between vitamin $\mathrm{E}$ and vitamin C. Nature 278: 737-738. [Crossref]

16. Chen R, Khormaee S, Eccleston ME, Slater NKH (2009) The role of hydrophobic amino acid grafts in the enhancement of membrane-disruptive activity of $\mathrm{pH}$-responsive pseudo-peptides. Biomaterials 30: 1954-1961. [Crossref]

17. LeBel CP, Ischiropoulos H, Bondy SC (1952) Evaluation of the probe 2',7'-dichlorofluorescin as an indicator of reactive oxygen species formation and oxidative stress. Chem Res Toxicol 5: 227-231. [Crossref]

18. Rastogi RP, Singh SP, Häder DP, Sinha RP (2010) Detection of reactive oxygen species (ROS) by the oxidant-sensing probe 2',7'-dichlorodihydrofluorescein diacetate in the cyanobacterium Anabaena variabilis PCC 7937. Biochem Biophys Res Commun 397: 603-607. [Crossref]

19. De Antoni GL, Pgrez P, Abraham A, Anon MC (1989) Trehalose, a Cryoprotectant for Lactobacillus bulgaricus. Cryobiology 26: 149-153.

20. Eroglu A, Toner M, Toth TL (2002) Beneficial effect of microinjected trehalose on the cryosurvival of human oocytes. Fertil Steril 77: 152-158. [Crossref]

21. Eroglu A, Lawitts JA, Toner M, Toth TL (2003) Quantitative microinjection of trehalose into mouse oocytes and zygotes, and its effect on development. Cryobiology 46: 121-134. [Crossref]

22. Alvarez JG, Storey BT (1992) Evidence for Increased Lipid Peroxidative Damage and Loss of Superoxide Dismutase Activity as a Mode of Sublethal Cryodamage to Human Sperm During Cryopreservation. J Androl 13: 232-241. [Crossref]

23. Neises B, Steglich W (1978) Simple Method for the Esterification of Carboxylic Acids. Angew Chemie Int Ed Engl 17: 522-524.

24. Seeman P, Cheng D, Iles GH (1973) Structure of membrane Holes in Osmotic and Saponin Hemolysis. J Cell Biol 56. [Crossref]

25. Sanvicens N, Pilar Marco M (2008) Multifunctional nanoparticles - properties and prospects for their use in human medicine. Trends Biotechnol 26: 425-433. [Crossref]

Copyright: (C2017 Chen A. This is an open-access article distributed under the terms of the Creative Commons Attribution License, which permits unrestricted use, distribution, and reproduction in any medium, provided the original author and source are credited. 